\title{
BMJ Open A randomised controlled trial to prevent smoking relapse among recently quit smokers enrolled in employer and health plan sponsored quitlines
}

Anna M McDaniel, ${ }^{1}$ Katrina A Vickerman, ${ }^{2}$ Timothy E Stump, ${ }^{3}$ Patrick O Monahan, ${ }^{3}$ Jeffrey L Fellows, ${ }^{4}$ Michael T Weaver, ${ }^{1}$ Beatriz H Carlini, ${ }^{6}$ Victoria L Champion, ${ }^{5}$ Susan M Zbikowski ${ }^{2}$

To cite: McDaniel AM, Vickerman KA, Stump TE, et al. A randomised controlled trial to prevent smoking relapse among recently quit smokers enrolled in employer and health plan sponsored quitlines. BMJ Open 2015;5: e007260. doi:10.1136/ bmjopen-2014-007260

- Prepublication history for this paper is available online. To view these files please visit the journal online (http://dx.doi.org/10.1136/ bmjopen-2014-007260).

Received 20 November 2014 Revised 15 April 2015 Accepted 8 May 2015

CrossMark

For numbered affiliations see end of article.

Correspondence to Dr Anna M McDaniel; annammcdaniel@ufl.edu

\section{ABSTRACT}

Objective: To test adding an interactive voice response (IVR)-supported protocol to standard quitline treatment to prevent relapse among recently quit smokers.

Design: Parallel randomised controlled trial with three arms: standard quitline, standard plus technology enhanced quitline with 10 risk assessments (TEQ-10), standard plus 20 TEQ assessments (TEQ-20).

Setting: Quit For Life (QFL) programme.

Participants: 1785 QFL enrolees through 19 employers or health plans who were $24+\mathrm{h}$ quit. Interventions: QFL is a 5-call telephone-based cessation programme including medications and webbased support. TEQ interventions included 10 or 20

IVR-delivered relapse risk assessments over 8 weeks with automated transfer to counselling for those at risk.

Main outcome measures: Self-reported 7-day and 30-day abstinence assessed at 6-month and 12-month post-enrolment (response rates: $61 \%$ and $59 \%$, respectively). Missing data were imputed.

Results: 1785 were randomised (standard $n=592$, TEQ-10 $n=602$, TEQ-20 $n=591$ ). Multiple imputationderived, intent-to-treat 30 -day quit rates $(95 \% \mathrm{Cl})$ at 6 months were $59.4 \%(53.7 \%$ to $63.8 \%)$ for standard, $62.3 \%$ ( $57.7 \%$ to $66.9 \%$ ) for TEQ- $10,59.4 \%(53.7 \%$ to $65.1 \%$ ) for TEQ-20 and 30 -day quit rates at 12 months were $61.2 \%$ (55.6\% to $66.8 \%$ ) for standard, $60.6 \%$ (56.0\% to $65.2 \%$ ) for TEQ- $10,54.9 \%$ $(49.0 \%$ to $60.9 \%)$ for TEQ-20. There were no significant differences in quit rates. $73.3 \%$ of TEQ participants were identified as at-risk by IVR assessments; on average, participants completed 0.41 IVR-transferred counselling calls. Positive risk assessments identified participants less likely (OR=0.56, $95 \% \mathrm{Cl} 0.42$ to 0.76 ) to be abstinent at 6 months.

Conclusions: Standard treatment was highly effective, with $61 \%$ remaining abstinent at 12 months using multiple imputation intent-to-treat (intent-to-treat missing=smoking quit rate: $38 \%$ ). TEQ assessments identified quitters at risk for relapse. However, adding IVR-transferred counselling did not yield higher quit

\section{Strengths and limitations of this study}

- First large randomised controlled trial to examine the use of interactive voice response (IVR) technology for prevention of smoking relapse.

- Large sample of participants recruited from employer and health plan quitline programmes serving tobacco users across the USA.

- Protocol used an innovative and potentially costeffective technology to connect those at risk for relapse to immediate counselling.

- We compared two risk assessment intensities over the first 8 weeks following successful abstinence.

- Although participants rated the IVR system positively, fewer accepted immediate risk-focused counselling than anticipated; thus, the increase in treatment exposure was small.

rates. Research is needed to determine if alternative designs can improve outcomes.

Trial registration number: NCT00888992.

Smoking continues to be the leading preventable cause of premature death and disease in the USA and worldwide. ${ }^{1}{ }^{2}$ Quitting tobacco is one of the most important steps a tobacco user can take to improve their health. ${ }^{3}$ Free publicly (state and government) and privately (eg, employer and health plan) funded telephone-based tobacco cessation quitlines are available throughout the USA and increasingly around the world to help tobacco users quit. With the advent of the Affordable Care Act in the USA, telephone-based tobacco cessation services offer an important resource to ensure that tobacco users have access to the empirically supported tobacco treatments that are required components of coverage. Although 
extensive evidence exists regarding the efficacy and costeffectiveness of tobacco cessation quitlines, ${ }^{4-6}$ as with other behavioural and pharmacological tobacco treatment options, little is known about how best to intervene with callers at the time of greatest risk for relapse.

A recent Cochrane Review concluded that previously tested behavioural relapse prevention interventions, which primarily included skills training or extended therapeutic contact, did not provide additional benefit over no treatment or intervention without a relapse prevention component. ${ }^{7}$ Survival studies have identified that the highest relapse risk, on average, is in the first 2 weeks after a quit attempt; however, there is considerable between-individual and within-individual variability in risk-related symptom patterns. ${ }^{8}$ Relapse prevention interventions may be most effective if provided at the time of relapse risk given the multifaceted and individualised nature of withdrawal and environmentally induced relapse risks over time. ${ }^{8}$

Interactive voice response (IVR) technology offers a resource-efficient solution for connecting tobacco users with relapse prevention interventions at the time of need, especially those already enrolled in phone-based tobacco cessation treatment. IVR is increasingly incorporated into some tobacco treatment protocols, ${ }^{9}{ }^{10}$ and has been used in chronic disease management, posthospital discharge management, medication compliance monitoring, and in a small number of addiction and depression self-help interventions. ${ }^{11}$ IVR has been piloted as a smoking relapse prevention tool following a single group counselling call and 12 weeks of varenicline, ${ }^{12}$ but was insufficiently powered to draw meaningful conclusions about the effectiveness of the nine-call and nurse call-back intervention. The purpose of this present study is to examine the effectiveness of augmenting standard multisession tobacco quitline treatment with repeated IVR assessments to monitor recent quitters' relapse risk, and transfer those at risk to a Quit Coach for a brief, focused, intervention targeting the identified relapse trigger. We hypothesised that smokers receiving IVR risk assessments during the first 2 months after quitting would be more likely to stay quit than those receiving standard quitline treatment, and that more frequent assessments (20 vs 10) would be more beneficial.

\section{METHODS}

\section{Study design}

In this parallel randomised controlled trial, tobacco users enrolled in the Quit For Life (QFL) programme who achieved abstinence for at least $24 \mathrm{~h}$ after their quit date were randomised into one of three study groups: (1) standard treatment, (2) Technology Enhanced Quitline-10 (TEQ-10): standard treatment plus 10 IVR-delivered relapse risk assessments which triggered a transfer to a Quit Coach for participants exceeding risk thresholds and (3) Technology Enhanced Quitline-20 (TEQ-20): same as the TEQ-10 condition except with 20 relapse risk assessments rather than 10. Participants were randomised using blocked randomisation to ensure similar group sizes throughout the study; every nine participants within each employer or health plan contract were randomly assigned with an allocation ratio for the three groups of 3:3:3. These random allocation tables were generated by the study management team and integrated into the QFL computer system where they were not accessible to coaches or participants. Once study eligibility was confirmed, the Quit Coach clicked a 'Randomise' button and the system automatically assigned the participant to the group listed in the next available cell of the allocation table. The planned and final sample size was 1785 and was determined a priori to achieve at least $80 \%$ power to detect 30 -day point prevalence quit rate differences of $8 \%$ between any pair of three arms for intent-to-treat (ITT) analyses, including the traditional penalised imputation approach in which non-responders to data collection are considered relapsed $^{13}$ and the state-of-the-art multiple imputation method $^{14}$ (assuming quit rates of $29.6 \%, 37.6 \%$ and $45.6 \%$ at 6 months and $26.6 \%, 34.6 \%$ and $42.6 \%$ at 12 months for standard care, TEQ-10 and TEQ-20, respectively; $\mathrm{n}=595$ ) and of $10 \%$ for a responder analysis between standard care and either intervention at 6 or 12 months (assuming quit rates of $52.1 \%$ standard care, 62.1\% TEQ-10, 62.1\% TEQ-20; assuming 25\% attrition, with 446 responders per arm). The primary analysis using ITT multiple imputation has slightly greater than $80 \%$ power because, like ITT using penalised imputation (missing=smoking), it uses all persons with baseline data. However, compared with penalised imputation, or responder-only analysis, multiple imputation ITT uses more information and produces results with greater power, less bias, and greater accuracy. ${ }^{13} 14$

Participants gave informed consent prior to study enrolment and received a US $\$ 25$ gift card for completed 6-month and 12-month follow-up surveys (potential for US\$50 total).

\section{Participants}

Tobacco users were eligible for the study if they had enrolled in the QFL programme between April 2010 and October 2012 through 1 of 19 employers or health plans who offered QFL as part of their benefits package. Because the purpose of this study was to examine the potential benefit of IVR assessments, and their triggering of counselling at the point of need to reduce relapse, tobacco users were screened for study criteria and invited to participate after reporting they had been quit for $24 \mathrm{~h}$ or more after their quit date. This typically occurred during their second or third call (their quit date call or the call after) of the five-call programme. Additional eligibility criteria included being English-speaking, 18 years of age or older, and having access to a touch-tone phone. Tobacco users were excluded if they exclusively used smokeless tobacco, were actively participating in another tobacco cessation programme, had previously enrolled in 
the QFL programme during the past 6 months, or had limited phone access (eg, incarceration).

Of the 3723 tobacco users who received the study offer, $1840(49.4 \%)$ agreed to participate and 1785 completed the baseline survey and were randomised to one of the three study groups (figure 1).

\section{The QFL programme}

QFL, operated by Alere Wellbeing (formerly Free \& Clear), has been commercially available for nearly 30 years, and its effectiveness and cost-effectiveness has been demonstrated in various studies. ${ }^{5}{ }^{15-19}$ The QFL programme includes five counselling calls (an initial assessment and planning call plus four additional proactive outbound calls from an expert Quit Coach). Quit Coaches are required to have a bachelor's degree in a related field, complete more than $200 \mathrm{~h}$ of training, and receive ongoing supervision and feedback. The call schedule and content is tailored to each participant's needs. The default call schedule includes the initial planning and assessment call, a quit date call (QDC), a quit date follow-up call 7 days after the QDC, and two additional follow-up calls that take place approximately every 3 weeks after the previous call. Participants are

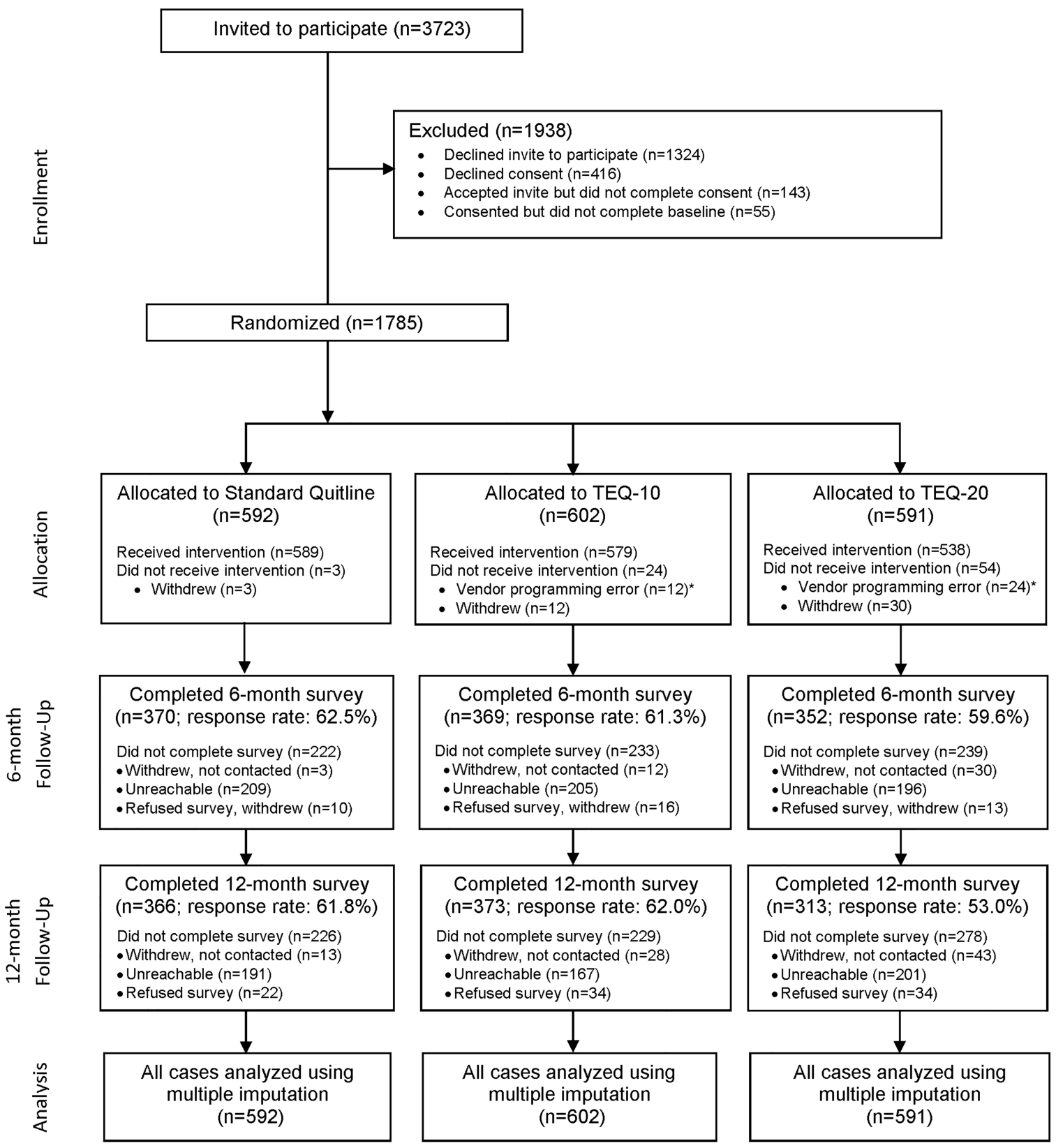

*36 participants did not receive all of their relapse risk assessments due to a programming error in the IVR code.

Figure 1 CONSORT Diagram. *36 participants did not receive all of their relapse risk assessments due to a programming error in the interactive voice response (IVR) code. 
also encouraged to call in for support as needed (ie, participant-initiated inbound calls). Calls vary in length: the planning and assessment call is $25-30 \mathrm{~min}$, while other calls are 15-20 min. Calls focus on creating a quit plan, developing problem-solving and coping skills, identifying social support, and using cessation medications to achieve and maintain abstinence. Participants may receive over-the-counter nicotine replacement products directly via mail (or other prescription cessation medications depending upon their cessation benefit with their employer or health plan). They also receive a printed quit guide, and access to Web Coach, an online interactive web-based programme designed to complement the phone programme.

\section{IVR intervention}

In the two TEQ groups, participants were contacted for relapse risk assessments through automated IVR calls over their first 8 weeks postquit. Two intensities of IVR monitoring were examined. TEQ-10 participants were contacted twice weekly for the first 2 weeks, then weekly for 6 weeks. TEQ-20 participants were contacted daily for the first 2 weeks, then weekly for 6 weeks.

An IVR service contractor programmed and delivered the risk assessments (approximately $5 \mathrm{~min}$ ), which included questions to identify relapse risk on five factors: lapses, cravings, negative affect, self-efficacy and motivation to remain quit. An algorithm was used to flag participants as 'at risk' if they answered any of the screening questions over an established threshold, based on previous research. ${ }^{20-22}$ Participants who exceeded the threshold were then transferred directly to a Quit Coach for a brief intervention (approximately $15 \mathrm{~min}$ ) specifically addressing the risk factor(s) that triggered their transfer. Intervention fidelity was monitored by study staff and investigators. Randomly sampled recordings of IVR-triggered telephone counselling sessions were rated for compliance with the study protocol (the primary criterion being whether the counsellor focused the call on identified relapse risk(s)) by two independent reviewers (a study staff member and a Quit Coach supervisor). Reviews revealed some Quit Coaches did not receive information about the risk factor that triggered a participant's transfer due to technological issues. These programming errors were corrected by the IVR vendor. In these circumstances, participants still received empirically based tobacco cessation support at the time of need. As part of quality monitoring, any protocol deviations were addressed with individual feedback from Quit Coach supervisors and ongoing training for all Quit Coaches.

\section{MEASURES}

\section{Baseline measures}

During registration and the first Quit Coach call, participants completed standard assessment questions regarding demographic characteristics (age, gender, health comorbidities) and tobacco use (tobacco type, time to first use, cigarettes per day, number of previous quit attempts, social contact with smokers). After study recruitment, participants completed an additional baseline measure assessing age, race, ethnicity, education, depressive symptoms (PHQ-2; 2-item measure ${ }^{21}$ ), and stress (Perceived Stress Scale; 4-item measure ${ }^{23}$ ).

\section{IVR-positive screens and program call completion}

The IVR system stored the number of relapse risk assessments participants answered, participants' responses to the relapse risk questions, and the number of IVR assessments on which a participant had a positive risk screen. Counselling calls completed as a result of a positive IVR screen and successful transfer to a Quit Coach (ie, IVR-transferred counselling calls) were tracked in addition to standard programme calls (5 planned calls plus participant-initiated inbound calls).

\section{Outcomes measured at 6 and 12 months}

At 6 and 12 months after randomisation, participants completed telephone interviews ${ }^{\mathrm{i}}$ administered by outcomes evaluators from the Indiana University Center for Survey Research who were blind to study condition to measure abstinence. All participants rated their satisfaction with the QFL service, and those in the TEQ groups were asked their opinions of the IVR system during the 6 -month survey. When participants could not be reached after repeated phone attempts, we also mailed a brief paper survey with only the smoking abstinence questions. Survey response rates were $61 \%$ and $59 \%$ at 6 and 12 months, respectively.

The primary study outcomes were quit rates at 6 and 12 months, defined as 7-day and 30-day self-reported point prevalence abstinence measured by the question, 'In the last [7/30] days, have you smoked a cigarette, even a puff?'. Time to relapse was examined as a secondary outcome and was identified using three questions: 'Since [end of two-week grace period], have you ever smoked at least a part of a cigarette on 7 days in a row?'; 'Since [end of two-week grace period], have you ever smoked any in each of two consecutive weeks (at least 1 day for 2 weeks in a row)?', and [if yes to either of the 2 previous questions] 'What was the first day of that 7-day or two-week period that you smoked?. ${ }^{16}$

\section{Statistical analyses}

Missing values on all 6-month and 12-month outcomes were imputed using the state-of-the-art imputation method called 'multiple imputation' ${ }^{24}$ with sequential regression. ${ }^{25}$ Multiple imputation allows all cases

${ }^{\mathrm{i}}$ Consistent with recommendation of the SRNT Subcommittee on Biochemical Verification (2000) for large scale (ie, >1000 participans), population-based studies with limited face-to-face contact and studies where the optimal data collection methods are through the mail, telephone, or internet, biochemical validation of abstinence was not obtained. 
$(\mathrm{N}=1785)$ to be included in the analysis. Two imputation models were performed: one for 7-day and one for 30-day point prevalence abstinence. With this approach, variables with no missing values served as independent variables. The variable with the least amount of missing data was imputed first, and used as the dependent variable. Once imputation was carried out, the next variable with the least amount of missing data was imputed using all variables with non-missing values as independent variables including the variable imputed on the previous step. This cycle continued until all missing values were imputed resulting in a series of regressions, hence the name sequential regression. Variables used to provide information for imputation (and to be imputed themselves if missing) included intervention group (no data missing), baseline characteristics listed in table 1 (with the exception of years of tobacco use and stress, due to high correlations with age and depression, respectively), and number of planned calls plus participant-initiated inbound counselling calls completed. These variables had a minimal amount of missing data with, at most, $3.5 \%$ missing for the motivation variable. Abstinence outcomes measured at both 6 and 12 months were included in each imputation model and had the greatest amount of missing data. Ten multiple-imputed data sets were generated and analysed.

Logistic regression models were used to model intervention efficacy on the 6-month and 12-month outcomes of abstinence, while adjusting for theoretically important potentially confounding baseline covariates. ${ }^{26}$ There were four main outcomes (7-day and 30-day point prevalence quit rates assessed at 6 and 12 months) and each was analysed with a separate logistic regression model. The multiple imputation quit rate results were our primary outcome metric. However, outcomes were reported using three methods: (1) for all survey respondents (responder analysis), (2) using penalised imputation reporting (assumes non-respondents are treatment failures) which is the traditional ITT analysis used in the smoking literature and (3) with results imputed for participants with missing data using the state-of-the-art multiple imputation method. Although ITT outcomes using penalised imputation are commonly reported in cessation trials, methodological research, including simulation studies, have shown that multiple imputation ITT uses more information and produces greater power, less bias, and more accuracy compared with simpler ad hoc methods for handling missing data such as traditional penalised imputation ITT, or responder-only analysis. ${ }^{13} 14$

Missing values for relapse date were imputed using the procedures described above. Using this relapse date and the baseline date, proportional hazards regression was used to examine factors associated with time to relapse. The variables representing number of calls (table 2) were generally skewed to the right with a large number of zeroes. These data were complete and did not require imputation. To compare study groups on these count variables, a generalised linear model with log link function and negative binomial distribution was used. Six-month satisfaction data were also analysed (without imputation) to determine levels of satisfaction with the QFL programme and IVR protocol. The satisfaction variable was not imputed since it was not a primary outcome. Satisfaction comparisons between intervention groups were made using a $\chi^{2}$ test. Mplus software for Windows (V.7.2) was used to generate imputed data and PROC MIANALYZE from SAS/STAT software (V.9.3) was used to pool results and analyse the imputed data sets. All other statistical comparisons were performed using SAS/BASE and SAS/STAT software for Windows (V.9.3).

\section{RESULTS}

\section{Participants}

Baseline characteristics across the three intervention groups are shown in table 1 . Overall, the average age of study participants was 43 years (range 18-85). A little over half $(54 \%)$ were women. A majority of the sample was Caucasian (83\%) and reported having a high school education or more $(62 \%)$. Just under a quarter of the sample reported having $\geq 1$ chronic comorbidity (22\%). The average number of reported cigarettes used per day was 17 (range 0-60) with about two-thirds of the sample reporting having had a cigarette within $30 \mathrm{~min}$ from the time they wake in the morning. About $20 \%$ of the sample reported 0 or one quit attempts, with a similar percentage reporting six or more quit attempts. A little over $60 \%$ reported using tobacco for 20 or more years, and about three-quarters reported having contact with smokers at work, home, or both. Overall, participants reported feeling motivated to quit smoking, with an average rating of 9 on a $1-10$ scale ( $1=$ low, $10=$ high). The average PHQ-2 depression score was 1.0 (possible range $0-6$, with high scores indicating higher levels of depression, ${ }^{21}$ and the average perceived stress score was 4.3 (possible range $0-16$, with higher scores indicating higher perceived stress. ${ }^{23}$ At the time of study offer, $56.5 \%$ of participants had been quit for 1-6 days, $40 \%$ had been quit for 7-29 days, and $3.5 \%$ had been quit for 30 days or more.

\section{Programme engagement and positive risk screens}

Programme engagement was measured as the number of counselling calls completed by participants: ${ }^{1}$ planned programme counselling calls, ${ }^{2}$ participant-initiated inbound calls, or ${ }^{3}$ IVR-transferred counselling calls (table 2). Overall, the average number of total calls completed was $3.9(\mathrm{SD}=1.7$; range $2-19)$. There was a statistical difference between groups; the TEQ-10 and TEQ-20 groups completed slightly more calls than the standard study group due to the additional IVR-transferred counselling calls they received after a positive screen $(p<0.001)$. There were no statistical differences between participant-initiated $(\mathrm{p}=0.91)$ or planned programme calls completed $(\mathrm{p}=0.81)$. Also, there was no statistical difference in IVR-transferred counselling calls between the TEQ-10 and TEQ-20 
Table 1 Baseline characteristics $(N=1785)$

\begin{tabular}{|c|c|c|c|c|}
\hline Variable & $\begin{array}{l}\text { Overall } \\
(\mathrm{N}=1785)\end{array}$ & $\begin{array}{l}\text { Standard } \\
(\mathrm{N}=592)\end{array}$ & $\begin{array}{l}\text { Standard plus } \\
\text { TEQ-10 }(\mathrm{N}=602)\end{array}$ & $\begin{array}{l}\text { Standard plus } \\
\text { TEQ-20 }(\mathrm{N}=591)\end{array}$ \\
\hline \multicolumn{5}{|l|}{ Demographics } \\
\hline Age, mean (SD) & $43.4(11.9)$ & 43.3 (12.2) & $44.0(11.5)$ & $43.0(12.0)$ \\
\hline \multicolumn{5}{|l|}{ Gender, N (\%) } \\
\hline Female & $967(54.2)$ & $321(54.2)$ & $326(54.1)$ & $320(54.2)$ \\
\hline Male & $818(45.8)$ & $271(45.8)$ & $276(45.9)$ & $271(45.8)$ \\
\hline \multicolumn{5}{|l|}{ Race, N (\%) } \\
\hline Caucasian & 1489 (83.4) & 497 (84.0) & 501 (83.2) & 491 (83.1) \\
\hline African-American & 204 (11.4) & $63(10.6)$ & $71(11.8)$ & $70(11.8)$ \\
\hline Other & $51(2.9)$ & $16(2.7)$ & $19(3.2)$ & $16(2.7)$ \\
\hline Missing & $43(2.3)$ & $16(2.7)$ & $11(1.8)$ & $14(2.4)$ \\
\hline \multicolumn{5}{|l|}{ Education, $\mathrm{N}(\%)$} \\
\hline High school or less & $667(37.4)$ & $229(38.7)$ & $225(37.4)$ & $213(36.0)$ \\
\hline More than high school & 1117 (62.6) & 363 (61.3) & $376(62.6)$ & $378(64.0)$ \\
\hline \multicolumn{5}{|l|}{ Comorbidities, N (\%) } \\
\hline Asthma & $176(9.9)$ & $62(10.5)$ & $61(10.1)$ & $53(9.0)$ \\
\hline COPD & $108(6.0)$ & $39(6.6)$ & $32(5.3)$ & $37(6.3)$ \\
\hline CAD & $75(4.2)$ & 27 (4.6) & $25(4.2)$ & $23(3.9)$ \\
\hline Diabetes & $138(7.7)$ & 53 (8.9) & $46(7.6)$ & $39(6.6)$ \\
\hline Any chronic comorbidity* & $395(22.1)$ & $136(23.0)$ & $136(22.6)$ & $123(20.8)$ \\
\hline \multicolumn{5}{|l|}{ Tobacco use } \\
\hline \multicolumn{5}{|l|}{ Time to first cigarette, $\mathrm{N}(\%), \min$} \\
\hline 5 & $539(30.2)$ & 165 (27.9) & $197(32.7)$ & $177(30.0)$ \\
\hline $6-30$ & $618(34.6)$ & $213(36.0)$ & $201(33.4)$ & $204(34.5)$ \\
\hline $31-60$ & $285(16.0)$ & 89 (15.0) & $101(16.8)$ & $95(16.1)$ \\
\hline$>60$ & 289 (16.2) & $105(17.7)$ & $88(14.6)$ & $96(16.2)$ \\
\hline Missing & $54(3.0)$ & $20(3.4)$ & $15(2.5)$ & 19 (3.2) \\
\hline Cigarettes per day, mean (SD) & $16.8(9.0)$ & $16.8(9.1)$ & $17.2(8.9)$ & $16.4(9.1)$ \\
\hline \multicolumn{5}{|l|}{ Number of quit attempts, $\mathrm{N}(\%)$} \\
\hline $0-1$ & $377(21.1)$ & $116(19.6)$ & $127(21.1)$ & $134(22.7)$ \\
\hline $2-5$ & $1033(57.9)$ & $371(62.7)$ & $335(55.7)$ & 327 (55.3) \\
\hline $6+$ & $347(19.4)$ & 97 (16.4) & $127(21.3)$ & $122(20.6)$ \\
\hline Missing & $28(1.6)$ & $8(1.4)$ & $12(2.0)$ & $8(1.4)$ \\
\hline \multicolumn{5}{|l|}{ Tobacco use $\geq 20$ years, $N(\%)$} \\
\hline Yes & $1098(61.5)$ & $361(61.0)$ & $383(63.6)$ & 354 (59.9) \\
\hline No & $671(37.6)$ & 227 (38.3) & $211(35.1)$ & $233(39.4)$ \\
\hline Missing & $16(0.9)$ & $4(0.7)$ & $8(1.3)$ & $4(0.7)$ \\
\hline \multicolumn{5}{|c|}{ Social contact with smokers, $\mathrm{N}(\%)$} \\
\hline No & $470(26.3)$ & $146(24.7)$ & $179(29.7)$ & $145(24.5)$ \\
\hline Work & $572(32.0)$ & 192 (32.4) & $188(31.2)$ & 192 (32.5) \\
\hline Home & $342(19.2)$ & 122 (20.6) & $104(17.3)$ & $116(19.6)$ \\
\hline Both work and home & 344 (19.3) & $112(18.9)$ & $111(18.4)$ & $121(20.5)$ \\
\hline Missing & 57 (3.2) & $20(3.4)$ & $20(3.3)$ & $17(2.9)$ \\
\hline \multicolumn{5}{|l|}{ Scale scores } \\
\hline Motivation to quit, mean (SD) & $9.1(1.3)$ & $9.0(1.3)$ & $9.0(1.2)$ & $9.1(1.2)$ \\
\hline Depression, mean (SD) & $1.0(1.3)$ & $1.0(1.3)$ & $1.0(1.3)$ & $1.0(1.3)$ \\
\hline Perceived stress, mean (SD) & $4.3(2.8)$ & $4.3(2.9)$ & $4.2(2.8)$ & $4.5(2.8)$ \\
\hline
\end{tabular}

${ }^{*}$ Defined as having asthma or COPD or CAD or diabetes.

CAD, coronary artery disease; COPD, chronic obstructive pulmonary disease; TEQ-10, Technology Enhanced Quitline-10; TEQ-20, Technology Enhanced Quitline-20.

groups $(\mathrm{p}=0.28)$. There was a statistical difference in the number of positive screens between the two intervention groups, with TEQ-20 having slightly more positive screens than TEQ-10 (on average, $1.52(\mathrm{SD}=1.66)$ for TEQ-10 and $1.82(\mathrm{SD}=1.99)$ for TEQ-20, $\mathrm{p}=0.003)$. The percentage of one or more positive screens was also larger in the TEQ-20 group ( $76 \%$ vs $70 \%$; $\mathrm{p}=0.03$ ).
Six-month and 12-month outcomes

Seven-day and 30-day point prevalence abstinence rates at 6-month and 12-month follow-up are shown in table 3. As aforementioned, outcomes are reported using three methods: (1) for all survey respondents (responder analysis), (2) using a crude ITT analysis based on penalised imputation reporting (assumes non-respondents are 


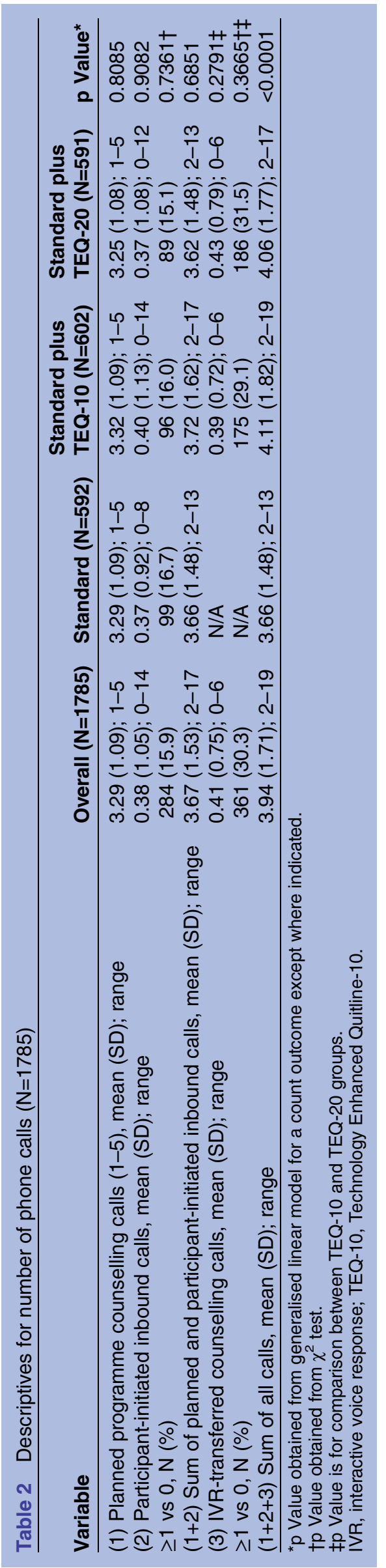

treatment failures) and (3) using state-of-the-art ITT based on multiple imputation for participants with missing data. Since we had missing data, we used the multiple imputation quit rate as our primary outcome.

ITT-penalised imputation quit rates at 12 months were significantly lower for the TEQ-20 group compared with the standard and TEQ-10 groups. However, there were no significant differences in responder or imputed quit rates at 6 or 12 months, or rates of relapse. The quit rates based on multiple imputation at 6 and 12 months ranged from $55 \%$ to $68 \%$ depending on intervention group, follow-up time point, and outcome measure.

Controlling for baseline characteristics, logistic regression models further illustrated no statistical difference between intervention groups for either primary outcome and either time point (table 4). A few baseline characteristics were statistically associated with the outcomes. Across the four models shown in table 4, a higher number of counselling calls, and being motivated to quit, were consistently associated with higher odds of being abstinent $($ all $\mathrm{p}<0.05)$. Depression also played a role in three of the models, with higher depression scores being associated with lower odds of being abstinent (all $\mathrm{p}<0.05)$. Older age resulted in higher odds of abstinence for 6 -month outcomes only (both $\mathrm{p}<0.01$ ). Having at least one comorbid chronic condition was associated with lower odds of abstinence in three of the models $($ all $\mathrm{p}<0.05)$.

\section{Time to relapse}

There was no significant association between time to relapse and randomisation group (table 5). Other factors related to time to relapse included number of counselling calls, having comorbid health condition, number of quit attempts, motivation to quit and depression. Higher number of counselling calls and higher levels of motivation to quit were associated with being less likely to relapse sooner $(\mathrm{HR}=0.87, \quad \mathrm{p}<0.001$; $\mathrm{HR}=0.84, \mathrm{p}<0.001$, respectively). By contrast, participants reporting higher levels of depression were more likely to relapse sooner $(\mathrm{HR}=1.07, \mathrm{p}=0.02)$. Compared to those with no chronic comorbidities, participants reporting one or more chronic comorbidities were more likely to relapse sooner $(\mathrm{HR}=1.29, \mathrm{p}=0.04)$. Those reporting six or more quit attempts were also more likely to relapse sooner compared to those with $0-1$ attempts $(\mathrm{HR}=1.32$, $\mathrm{p}=0.04)$.

\section{Using IVR to identify risk}

Figure 2 presents quit outcomes for TEQ-10 and TEQ-20 groups (separately in panel A and combined in panel B) according to their positive screen status $(\geq 1$ positive screen vs no positive screen); $70.4 \%$ in TEQ-10 and $76.1 \%$ in TEQ-20 had $\geq 1$ positive screen during the study. Compared with participants who screened positive in either TEQ group, participants who did not screen positive were more likely to be abstinent at $6(\mathrm{OR}=1.69$, $\mathrm{p}=0.01$ for 7 -day point prevalence; $\mathrm{OR}=1.77, \mathrm{p} \leq 0.001$ for 
Table 3 Estimates of abstinence at 6 and 12 months, $N=1785$

\begin{tabular}{|c|c|c|c|c|c|c|c|c|}
\hline \multirow[b]{2}{*}{ Intervention group } & \multicolumn{4}{|c|}{ 6-Month survey outcomes } & \multicolumn{4}{|c|}{ 12-Month survey outcomes } \\
\hline & $\mathbf{N}$ & $\begin{array}{l}\text { Did not smoke } \\
\text { in last } 7 \text { days }\end{array}$ & $\mathbf{N}$ & $\begin{array}{l}\text { Did not smoke } \\
\text { in the last } 30 \text { days }\end{array}$ & $\mathbf{N}$ & $\begin{array}{l}\text { Did not smoke } \\
\text { in last } 7 \text { days }\end{array}$ & $\mathbf{N}$ & $\begin{array}{l}\text { Did not smoke } \\
\text { in the last } 30 \text { days }\end{array}$ \\
\hline \multicolumn{9}{|l|}{ Responders* } \\
\hline Standard & 368 & $66.0(61.2$ to 70.9$)$ & 368 & 60.6 (55.6 to 65.6$)$ & 363 & 65.3 (60.4 to 70.2$)$ & 362 & 61.6 (56.6 to 66.6$)$ \\
\hline Standard plus TEQ-10 & 368 & 69.6 (64.9 to 74.3$)$ & 368 & $65.2(60.4$ to 70.1$)$ & 370 & $67.0(62.2$ to 71.8$)$ & 371 & 63.1 (58.2 to 68.0$)$ \\
\hline Difference compared to standard & & $\begin{array}{l}3.5(-3.2 \text { to } 10.3) \\
p=0.3051\end{array}$ & & $\begin{array}{l}4.6(-2.4 \text { to } 11.6) \\
p=0.1946\end{array}$ & & $\begin{array}{l}1.7(-5.1 \text { to } 8.6) \\
\mathrm{p}=0.6191\end{array}$ & & $\begin{array}{l}1.5(-5.5 \text { to } 8.5) \\
\mathrm{p}=0.6812\end{array}$ \\
\hline Standard plus TEQ-20 & 352 & $67.3(62.4$ to 72.2$)$ & 352 & 61.1 (56.0 to 66.2$)$ & 310 & $62.6(57.2$ to 68.0$)$ & 311 & $56.6(51.1$ to 62.1$)$ \\
\hline \multirow[t]{2}{*}{ Difference compared to standard } & & $1.3(-5.6$ to 8.2$)$ & & $0.5(-6.6$ to 7.6$)$ & & $-2.7(-10.0$ to 4.6$)$ & & $-5.0(-12.5$ to 2.4$)$ \\
\hline & & $\mathrm{p}=0.7121$ & & $\mathrm{p}=0.8947$ & & $\mathrm{p}=0.4655$ & & $\mathrm{p}=0.1871$ \\
\hline \multicolumn{9}{|c|}{ Intent-to-treat penalised imputation (missing=smoking) $†$} \\
\hline Standard & 592 & 41.1 (37.1 to 45.0$)$ & 592 & 37.6 (33.8 to 41.6$)$ & 592 & 40.0 (36.1 to 44.0$)$ & 592 & 37.7 (33.8 to 41.6$)$ \\
\hline Standard plus TEQ-10 & 602 & 42.5 (38.6 to 46.5$)$ & 602 & 39.9 (36.0 to 43.8$)$ & 602 & $41.2(37.3$ to 45.1$)$ & 602 & 38.9 (35.0 to 42.8$)$ \\
\hline \multirow[t]{2}{*}{ Difference compared to standard } & & $1.5(-4.1$ to 7.1$)$ & & $2.2(-3.3$ to 7.7$)$ & & $1.2(-4.4$ to 6.7$)$ & & $1.2(-4.3$ to 6.7$)$ \\
\hline & & $\mathrm{p}=0.6047$ & & $\mathrm{p}=0.4357$ & & $\mathrm{p}=0.6827$ & & $\mathrm{p}=0.6693$ \\
\hline Standard plus TEQ-20 & 591 & 40.1 (36.2 to 44.1$)$ & 591 & 36.4 (32.5 to 40.3$)$ & 591 & 32.8 (29.0 to 36.6$)$ & 591 & 29.8 (26.1 to 33.5$)$ \\
\hline Difference compared to standard & & $\begin{array}{l}-1.0(-6.5 \text { to } 4.7) \\
p=0.7405\end{array}$ & & $\begin{array}{l}-1.3(-6.8 \text { to } 4.2) \\
p=0.6459\end{array}$ & & $\begin{array}{l}-7.2(-12.7 \text { to }-1.7) \\
p=0.0100\end{array}$ & & $\begin{array}{l}-7.9(-13.3 \text { to }-2.5) \\
p=0.0041\end{array}$ \\
\hline \multicolumn{9}{|l|}{ Multiple imputation $\ddagger$} \\
\hline Standard & 592 & 64.2 (59.5 to 68.9$)$ & 592 & 59.4 (53.7 to 63.8$)$ & 592 & 63.3 (58.4 to 68.4$)$ & 592 & $61.2(55.6$ to 66.8$)$ \\
\hline Standard plus TEQ-10 & 602 & $67.8(63.0$ to 72.7$)$ & 602 & $62.3(57.7$ to 66.9$)$ & 602 & $65.1(60.3$ to 69.9$)$ & 602 & 60.6 (56.0 to 65.2$)$ \\
\hline Difference compared to standard & & $\begin{array}{l}3.6(-3.3 \text { to } 10.5) \\
p=0.2980\end{array}$ & & $\begin{array}{l}3.6(-3.2 \text { to } 10.4) \\
p=0.2969\end{array}$ & & $\begin{array}{l}1.7(-4.4 \text { to } 7.7) \\
p=0.5831\end{array}$ & & $\begin{array}{l}-0.6(-7.6 \text { to } 6.4) \\
p=0.8647\end{array}$ \\
\hline Standard plus TEQ-20 & 591 & 64.7 (59.2 to 70.2$)$ & 591 & $59.4(53.7$ to 65.1$)$ & 591 & $60.9(56.4$ to 65.5$)$ & 591 & $54.9(49.0$ to 60.9$)$ \\
\hline \multirow[t]{2}{*}{ Difference compared to standard } & & $0.5(-7.0$ to 8.0$)$ & & $0.7(-6.4$ to 7.8$)$ & & $-2.5(-9.4$ to 4.5$)$ & & $-6.3(-14.9$ to 2.3$)$ \\
\hline & & $\mathrm{p}=0.8982$ & & $\mathrm{p}=0.8468$ & & $\mathrm{p}=0.4808$ & & $\mathrm{p}=0.1471$ \\
\hline $\begin{array}{l}\text { Percentage of participants reporting abst } \\
\text { *Estimates were obtained among particip } \\
\text { †Estimates were obtained assuming part } \\
\text { †Estimates were obtained by pooling res } \\
\text { TEQ-10, Technology Enhanced Quitline- }\end{array}$ & 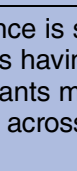 & $\begin{array}{l}\mathrm{n} \text { in table above with } \\
\text { mpleted a 6-month o } \\
\mathrm{g} \text { a } 6 \text {-month or } 12-\mathrm{mc} \\
\text { multiply imputed data }\end{array}$ & 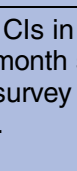 & $\begin{array}{l}\text { entheses. } p \text { Values ob } \\
\text { ey. } \\
\text { not abstinent. }\end{array}$ & & & & \\
\hline
\end{tabular}

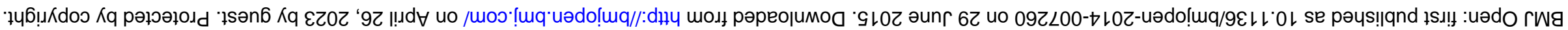


Table 4 ORs from adjusted logistic models of multiple imputation quit rates at 6 and 12 months, N=1785

\begin{tabular}{|c|c|c|c|c|}
\hline \multirow[b]{2}{*}{ Variable } & \multicolumn{2}{|c|}{ 6-Month survey outcomes } & \multicolumn{2}{|c|}{ 12-Month survey outcomes } \\
\hline & $\begin{array}{l}\text { Did not smoke } \\
\text { in the last } 7 \text { days }\end{array}$ & $\begin{array}{l}\text { Did not smoke } \\
\text { in the last } 30 \text { days }\end{array}$ & $\begin{array}{l}\text { Did not smoke } \\
\text { in the last } 7 \text { days }\end{array}$ & $\begin{array}{l}\text { Did not smoke } \\
\text { in the last } 30 \text { days }\end{array}$ \\
\hline \multicolumn{5}{|l|}{ Randomisation groups } \\
\hline $\begin{array}{l}\text { Standard plus TEQ-10 vs } \\
\text { standard }\end{array}$ & $1.15(0.83$ to 1.59$)$ & $1.14(0.85$ to 1.54$)$ & $1.07(0.82$ to 1.41$)$ & $0.96(0.71$ to 1.30$)$ \\
\hline $\begin{array}{l}\text { Standard plus TEQ-20 vs } \\
\text { standard }\end{array}$ & $1.00(0.71$ to 1.42$)$ & $1.01(0.74$ to 1.37$)$ & $0.87(0.64$ to 1.19$)$ & 0.75 (0.52 to 1.09$)$ \\
\hline \multicolumn{5}{|c|}{ Number of programme counselling calls } \\
\hline Planned+participant-initiated & $1.10(1.02 \text { to } 1.18)^{*}$ & $1.10(1.02 \text { to } 1.19)^{*}$ & $1.12(1.02 \text { to } 1.22)^{\star}$ & $1.10(1.02 \text { to } 1.19)^{\star}$ \\
\hline \multicolumn{5}{|l|}{ Demographics } \\
\hline \multicolumn{5}{|l|}{ Gender } \\
\hline Female vs male & $0.92(0.70$ to 1.21$)$ & $1.00(0.80$ to 1.25$)$ & $1.17(0.91$ to 1.49$)$ & $1.16(0.92$ to 1.46$)$ \\
\hline Age & $1.02(1.01 \text { to } 1.03)^{\star \star \star}$ & $1.02(1.00 \text { to } 1.03)^{\star *}$ & 1.01 (0.99 to 1.02$)$ & 1.01 (0.99 to 1.02$)$ \\
\hline \multicolumn{5}{|c|}{ 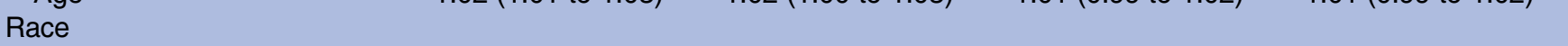 } \\
\hline $\begin{array}{l}\text { African-American vs } \\
\text { Caucasian }\end{array}$ & $1.32(0.89$ to 1.96$)$ & $1.41(0.91$ to 2.21$)$ & $1.16(0.75$ to 1.80$)$ & $1.12(0.72$ to 1.76$)$ \\
\hline Other vs Caucasian & $0.73(0.34$ to 1.56$)$ & $0.72(0.32$ to 1.66$)$ & 1.09 (0.55 to 2.16$)$ & $0.98(0.53$ to 1.81$)$ \\
\hline \multicolumn{5}{|l|}{ Education } \\
\hline $\begin{array}{l}\text { High school or less vs more } \\
\text { than high school }\end{array}$ & $1.12(0.84$ to 1.50$)$ & 1.05 (0.77 to 1.43$)$ & $0.86(0.65$ to 1.13$)$ & $1.01(0.76$ to 1.33$)$ \\
\hline \multicolumn{5}{|l|}{ Comorbidities } \\
\hline Any chronic comorbidity & $0.69(0.51 \text { to } 0.94)^{*}$ & $0.65(0.46 \text { to } 0.92)^{*}$ & $0.72(0.53 \text { to } 0.98)^{*}$ & $0.76(0.56$ to 1.03$)$ \\
\hline \multicolumn{5}{|l|}{ Tobacco use } \\
\hline Cigarettes per day & $0.98(0.97 \text { to } 0.99)^{*}$ & $0.99(0.97$ to 1.00$)$ & $0.98(0.97$ to 1.00$)$ & 0.99 (0.98 to 1.00$)$ \\
\hline \multicolumn{5}{|l|}{ Time to first cigarette } \\
\hline 5 min vs all others & $0.92(0.68$ to 1.24$)$ & $1.10(0.82$ to 1.48$)$ & $0.88(0.64$ to 1.20$)$ & 0.85 (0.65 to 1.12$)$ \\
\hline \multicolumn{5}{|l|}{ Number of quit attempts } \\
\hline $2-5$ vs $0-1$ & $0.96(0.69$ to 1.34$)$ & $0.98(0.75$ to 1.29$)$ & $0.80(0.58$ to 1.10$)$ & $0.93(0.69$ to 1.24$)$ \\
\hline $6+$ vs $0-1$ & 0.88 (0.57 to 1.35$)$ & $0.85(0.56$ to 1.28$)$ & $0.68(0.47 \text { to } 0.99)^{*}$ & 0.79 (0.55 to 1.13$)$ \\
\hline \multicolumn{5}{|l|}{ Social contact with smokers } \\
\hline Work or home vs all others & $0.74(0.56 \text { to } 0.97)^{*}$ & $0.95(0.73$ to 1.24$)$ & $0.98(0.74$ to 1.30$)$ & $0.89(0.68$ to 1.17$)$ \\
\hline \multicolumn{5}{|l|}{ Scale scores } \\
\hline Motivation to quit & $1.24(1.12 \text { to } 1.36)^{\star * *}$ & $1.24(1.11 \text { to } 1.39)^{\star \star *}$ & $1.22(1.11 \text { to } 1.34)^{\star \star \star}$ & $1.23(1.12 \text { to } 1.35)^{\star \star *}$ \\
\hline Depression & $0.87(0.78 \text { to } 0.96)^{\star \star}$ & $0.88(0.80 \text { to } 0.97)^{\star *}$ & $0.94(0.83$ to 1.06$)$ & $0.89(0.80 \text { to } 0.99)^{\star}$ \\
\hline
\end{tabular}

30-day point prevalence) and 12 months ( $\mathrm{OR}=1.47$, $\mathrm{p}=0.03$ for 7-day point prevalence; $\mathrm{OR}=1.50, \mathrm{p}=0.01$ for 30-day point prevalence).

\section{Satisfaction with QFL and the IVR protocol}

Satisfaction was measured at 6 months after enrolment. Participants completing the 6-month survey $(n=1091$, $61.1 \%$ ) were generally satisfied with the overall QFL programme and the counselling they received $(96 \%$ and $98 \%$, respectively, were somewhat satisfied, satisfied, or very satisfied at 6 months). A similar percentage (98\%) would recommend the programme to others. There were no differences in QFL programme satisfaction between the three study groups. Compared to TEQ-10 participants, those receiving TEQ-20 were more likely to report the number of relapse risk assessments were too many $(36 \%$ vs $23 \%, \mathrm{p} \leq 0.001)$ and also were marginally more likely to agree that the risk assessments took too much time ( $28 \%$ vs $23 \%, \mathrm{p}=0.09)$. The majority of participants reported it was easy to answer questions through the IVR system (95\%), that the system was helpful $(87 \%)$, and that they would recommend the IVR system to others trying to stay quit $(86 \%)$, with no significant differences between the TEQ-10 and TEQ-20 groups.

\section{DISCUSSION}

In this randomised controlled trial investigating the use of an automated, IVR-supported protocol to identify risk and prevent smoking relapse among recently quit tobacco users enrolled in the QFL programme, there were no differences in tobacco abstinence rates between the control (standard QFL) and technology-enhanced (IVR-delivered risk assessments and transfer for counselling) groups at 6-month and 12-month follow-up. Although the risk assessment and transfer protocols did 
Table 5 HRs from adjusted proportional hazard models of time to relapse, $\mathrm{N}=1785$

\begin{tabular}{|c|c|c|}
\hline Variable & HR (95\% Cl) & p Value \\
\hline \multicolumn{3}{|l|}{ Randomisation groups } \\
\hline Standard plus TEQ-10 vs standard & 0.85 (0.69 to 1.04$)$ & 0.1171 \\
\hline Standard plus TEQ-20 vs standard & $1.05(0.85$ to 1.31$)$ & 0.6282 \\
\hline \multicolumn{3}{|l|}{ Number of programme counselling calls } \\
\hline Planned+participant-initiated & $0.87(0.82 \text { to } 0.92)^{\star \star *}$ & $<0.0001$ \\
\hline \multicolumn{3}{|l|}{ Demographics } \\
\hline \multicolumn{3}{|l|}{ Gender } \\
\hline Female vs male & $0.90(0.75$ to 1.09$)$ & 0.2838 \\
\hline Age & $1.00(0.99$ to 1.01$)$ & 0.5278 \\
\hline \multicolumn{3}{|l|}{ Race } \\
\hline African-American vs Caucasian & $0.97(0.75$ to 1.26$)$ & 0.8400 \\
\hline Other vs White & $1.11(0.67$ to 1.84$)$ & 0.6762 \\
\hline \multicolumn{3}{|l|}{ Education } \\
\hline High school or less vs more than high school & $1.06(0.87$ to 1.30$)$ & 0.5368 \\
\hline \multicolumn{3}{|l|}{ Comorbidities } \\
\hline Any chronic comorbidity & $1.29(1.01 \text { to } 1.64)^{*}$ & 0.0430 \\
\hline \multicolumn{3}{|l|}{ Tobacco use } \\
\hline Cigarettes per day & 1.01 (0.99 to 1.02$)$ & 0.0639 \\
\hline \multicolumn{3}{|l|}{ Time to first cigarette } \\
\hline $5 \mathrm{~min}$ vs all others & $1.08(0.88$ to 1.34$)$ & 0.4430 \\
\hline \multicolumn{3}{|l|}{ Number of quit attempts } \\
\hline $2-5$ vs $0-1$ & $1.14(0.90$ to 1.43$)$ & 0.2657 \\
\hline $6+$ vs $0-1$ & $1.32(1.02 \text { to } 1.72)^{*}$ & 0.0345 \\
\hline \multicolumn{3}{|l|}{ Social contact with smokers } \\
\hline Work or home vs all others & $0.99(0.80$ to 1.24$)$ & 0.9713 \\
\hline \multicolumn{3}{|l|}{ Scale scores } \\
\hline Motivation to quit & $0.84(0.79 \text { to } 0.90)^{\star \star \star}$ & $<0.0001$ \\
\hline Depression & $1.07(1.01 \text { to } 1.13)^{\star}$ & 0.0198 \\
\hline
\end{tabular}

not yield better abstinence rates, the IVR-delivered screenings did successfully identify participants who were less likely to be quit at follow-up. Participants who did not screen positive during the relapse risk assessments were $77 \%$ more likely to be abstinent at 6 -month follow-up. We tested two risk screening and transfer protocols of varying intensities. The more intensive screening protocol with 20 IVR-delivered assessments did not demonstrate any benefit over the 10-assessment group, and the TEQ-20 respondents were more likely to indicate there were too many IVR assessments. In addition, we explored whether the TEQ intervention was more effective for certain populations in this study with regard to quit outcomes, by testing interaction effects, but did not identify any significant moderating relationships.

There are several possible explanations for why the intervention did not reduce relapse rates. The TEQ interventions were designed to immediately transfer participants who met risk thresholds for tobacco counselling to target the identified relapse risk. About three-quarters $(73.3 \%)$ of the TEQ participants were identified as being at risk at least one time over the 8 -week intervention period. Having a positive screen was predictive of relapse; however, it is possible that other risk factors are more predictive of risk than those included in the assessment. Also, participants identified as at risk by the IVR assessments and automatically transferred to a coach failed to remain on the line to receive cessation counselling at the anticipated rate. That is, less than one third of those in the TEQ arms completed an IVR-transferred counselling call and $41.3 \%$ of those with a positive risk screen completed one or more IVR-transferred counselling calls, resulting in participants in the TEQ conditions completing only 0.41 calls, on average, more than the planned programme calls from the standard programme. Thus, it is possible that we failed to observe an effect because we did not increase the actual treatment exposure much above the base rate of calls. It remains unclear why some callers did not accept these additional calls when the satisfaction data showed positive perceptions of the IVR automated assessment system (87\% felt it was helpful when they need to talk to a Quit Coach; $86 \%$ would recommend). Most callers were still receiving planned standard programme calls at the time of their risk assessments; perhaps callers who had recently spoken to a coach did not feel they needed another call at that time. Future studies should assess reasons for lack of 


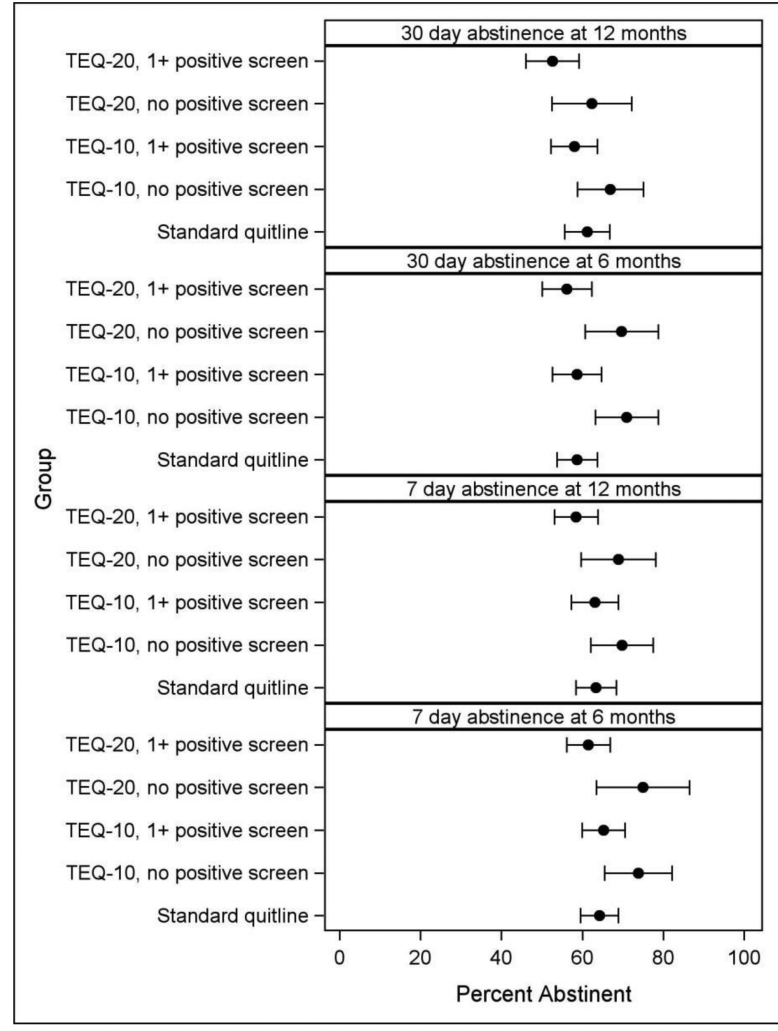

Panel A

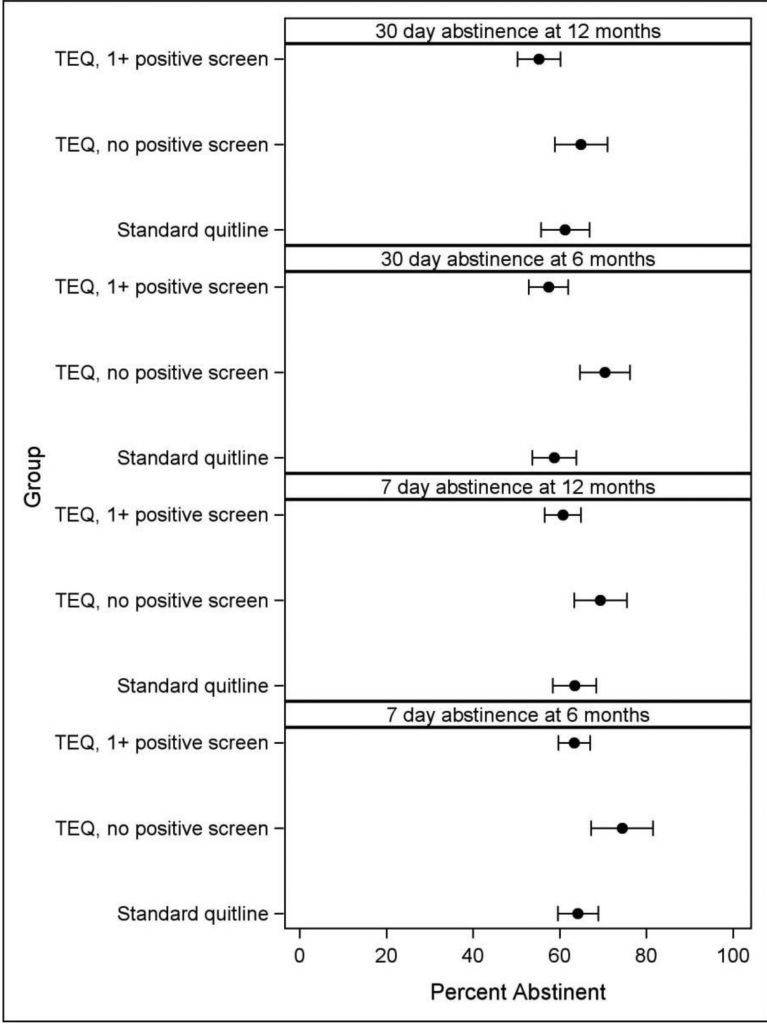

Panel B

Figure 2 Abstinence rates for intervention and positive screen groups, $\mathrm{N}=1785$. Estimates for each model were obtained by combining results across 10 multiple-imputed data sets. Error bars indicate $95 \%$ Cls. (A) Displays results for the TEQ-10 and TEQ-20 groups separately. (B) Displays data for the two TEQ-groups combined. '1+ positive screen' is defined as exceeding the predetermined risk threshold on any of the IVR assessment questions (topics: lapses, cravings, negative effect, self-efficacy, and motivation to remain quit) on any IVR assessment call. IVR, interactive voice response; TEQ-10, Technology Enhanced Quitline-10; TEQ-20, Technology Enhanced Quitline-20.

counselling uptake and consider an automated system that would allow participants to schedule a call in the near future or would initiate outbound calls to participants identified as at risk who do not immediately transfer to a counsellor.

The study had high quit outcomes, in spite of the fact that the TEQ intervention did not significantly improve outcomes. The $61 \%$ respondent and $38 \%$ ITT penalised imputation (missing=smoking) 30-day point prevalence quit rates at 6 months in the standard treatment group indicated that tobacco users who receive a multiple-call phone counselling programme, have access to cessation medication benefits, and successfully achieve at least a $24 \mathrm{~h}$ abstinence period early in treatment do quite well. These quit rates are high compared to studies focusing on all commercial quitline enrolees (eg, 34.3-41.1\% responder quit rate at 6 months ${ }^{27}{ }^{28}$ ). The relatively strong performance and engagement in standard treatment for this study may have made achieving improvements with a low intensity relapse prevention intervention even more challenging. This finding also aligns with previous recommendations that increasing quit attempts may be one of the most important factors for increasing cessation at the population level, ${ }^{29}$ and demonstrates the importance of achieving at least $24 \mathrm{~h}$ of abstinence.

More counselling calls completed, higher motivation to quit, lower depression scores, and not having chronic health condition were consistently related to greater likelihood of being abstinent at follow-up. These variables were also associated with time to relapse; additionally, callers with a greater number of previous quit attempts $(6+)$ were more likely to relapse sooner. These findings are consistent with previous quitline studies which have demonstrated the importance of programme engagement (ie, number of calls completed ${ }^{15} 3031$ ). Callers in this study had a high motivation to quit (9 out of 10 , on average); a previous study has linked high motivation to quit with making a quit attempt but not with likelihood of remaining quit. ${ }^{32}$ Moreover, previous work has identified that callers with depressive symptoms ${ }^{33}$ and chronic health conditions ${ }^{31}$ may have more difficulty quitting. Dependence level (time to first use and cigarettes per day) was not consistently related to outcomes in this study, which differs from previous literature that has often linked baseline dependence and outcomes. ${ }^{31} 32$

Compared with other behavioural relapse prevention research, strengths of this study included a relatively large 
sample size, use of an innovative and potentially costeffective technology that could be disseminated to large populations if shown to be effective, and attempting to connect those at risk to immediate counselling. Most previous behavioural relapse prevention studies have focused on skills training and have not yielded significant effects. ${ }^{7}$ As noted by Hajek et $a l^{7}{ }^{7}$ future relapse prevention research efforts may be best applied in examining new and alternative approaches to those previously studied. One recent small pilot study examined extended IVR assessments following varenicline treatment, and also did not find significant effects for the extended IVR group. ${ }^{12}$ That article did not report on whether those who screened positive during IVR assessments were more likely to have returned to smoking at follow-up, and likely had too small of sample size to meaningfully do so. Additionally, the McNaughton et al $\mathrm{s}^{12}$ study included an intensive IVR contact schedule with 80 contacts over 40 weeks; low IVR assessment completion rates $(37 \%$ of IVR calls and $16 \%$ of assessments were completed) in that study align with our findings that more contact is not necessarily better. Identifying the most effective and costeffective number of contacts should continue to be a focus in future studies using IVR technology.

The response rates to our 6-month and 12-month outcome surveys were $61 \%$ and $59 \%$, respectively, which may limit the validity of our findings. Multiple imputation, a preferred method for ITT analysis, was used to reduce potential non-response bias, and the findings from these analyses agreed closely with results from a sensitivity analysis using respondent-only data. Another potential limitation to our study design is the lack of biochemical verification of abstinence, particularly in light of the high reported abstinence rates. However, our study procedures were consistent with recommendations of the SRNT Subcommittee on Biochemical Verification, ${ }^{34}$ and the results should be considered with respect to this. Additionally, we have no reason to believe there was a systematic bias in reporting abstinence across groups. Third, the protocol aimed for IVR-triggered counselling calls to focus on the specific risk factor(s) identified by the IVR assessment; all participants received empirically based support at the time of need, but not all calls were focused on the identified risk factor(s). Finally, as with other research on prevention of smoking relapse, the time frame for intervention and data collection poses limitations. The IVR-delivered risk assessments in this study were limited to the first 8 weeks following successful abstinence. Although previous research has identified the first weeks as being the greatest risk for relapse, callers also relapse at later dates. Ongoing plans for prevention of relapse, or risk intervention, may be needed; future research may be able to identify those in need of ongoing relapse risk monitoring and intervention.

Augmenting tobacco quitline treatment with IVR-delivered relapse risk screening assessments was acceptable to participants, and successfully identified recently quit tobacco users who may be in need of more intensive or different intervention. More research is needed to identify how to engage at-risk participants in additional treatment. Using a technology-based screening for relapse risk to connect recent tobacco users to cessation counselling at the time of risk could offer a scalable, cost-effective approach for delivering tailored services to those in need, if future research can identify the appropriate services to deliver at that time of need.

\section{Author affiliations}

${ }^{1}$ College of Nursing, University of Florida, Gainesville, Florida, USA

${ }^{2}$ Alere Wellbeing, Seattle, Washington, USA

${ }^{3}$ Department of Biostatistics, Indiana University, School of Medicine, Indianapolis, Indiana, USA

${ }^{4}$ Kaiser Permanente Center for Health Research, Portland, Oregon, USA

${ }^{5}$ Indiana University School of Nursing, Indianapolis, Indiana, USA

${ }^{6}$ Alcohol and Drug Abuse Institute, University of Washington, Seattle, Washington, USA

Acknowledgements The authors would like to thank the following individuals who significantly contributed to this project: Mark Campbell, Renee Stratton and Barbara Cerutti who provided essential study management, implementation support, and monitoring of data and procedures; Freddie Harris for managing the study database; the employers and health plans who agreed to take part in the study; the coaches who delivered services; and the quitline callers who agreed to participate in the study.

Contributors AMM conceived of and designed the study protocol, interpreted the data, and revised the paper. AMM is the guarantor. KAV interpreted the data and drafted and revised the paper. POM and MTW oversaw the statistical analysis plan, interpreted the data, and revised the paper. TES analysed and interpreted the data, drafted the results and tables/figures, and revised the paper. JLF interpreted the data and revised the paper. SMZ and BHC designed and oversaw the study protocol, interpreted the data, and revised the paper. VLC contributed to the study design, interpreted the data and revised the paper. TES and POM had access to all data in the study and can take responsibility for the integrity of the data.

Funding The study was funded by the National Institutes for Health (National Cancer Institute grant number R01 CA138936-03) from the United States Department of Health and Human Services.

Competing interests KAV, BHC, and SMZ declare employment at Alere Wellbeing, the provider of quitline services in this study.

Ethics approval Indiana University Institutional Review Board approved the study protocol (approval ID number 1009001892)

Provenance and peer review Not commissioned; externally peer reviewed.

Data sharing statement The full trial protocol is available on request, from the lead author.

Open Access This is an Open Access article distributed in accordance with the Creative Commons Attribution Non Commercial (CC BY-NC 4.0) license, which permits others to distribute, remix, adapt, build upon this work noncommercially, and license their derivative works on different terms, provided the original work is properly cited and the use is non-commercial. See: http:// creativecommons.org/licenses/by-nc/4.0/

\section{REFERENCES}

1. World Health Organization. WHO global report on mortality attributable to tobacco. Geneva, Switzerland: World Health Organization, 2012.

2. US Department of Health and Human Services, Centers for Disease Control and Prevention, National Center for Chronic Disease Prevention and Health Promotion, Office on Smoking and Health. The health consequences of smoking - 50 years of progress: a report of the surgeon general. 2014, Report No: 17. 
3. U.S. Department of Health and Human Services, Centers for Disease Control and Prevention, National Center for Chronic Disease Prevention and Health Promotion, Office on Smoking and Health. What to tell your patients about smoking-a report of the Surgeon General: how tobacco smoke causes disease. 2010.

4. Fiore MC, Jaen CR, Baker TB, et al. Treating tobacco use and dependence: 2008 update. Rockville, MD: US Department of Health and Human Services, 2008.

5. Hollis JF, McAfee TA, Fellows JL, et al. The effectiveness and cost effectiveness of telephone counselling and the nicotine patch in a state tobacco quitline. Tob Control 2007;16(Suppl 1):i53-9.

6. Zhu SH, Anderson CM, Tedeschi GJ, et al. Evidence of real-world effectiveness of a telephone quitline for smokers. $N$ Engl J Med 2002;347:1087-93.

7. Hajek $\mathrm{P}$, Stead LF, West $\mathrm{R}$, et al. Relapse prevention interventions for smoking cessation. Cochrane Database Syst Rev 2013;8: CD003999.

8. Piasecki TM. Relapse to smoking. Clin Psychol Rev 2006;26:196-215.

9. McDaniel AM, Benson PL, Roesener GH, et al. An integrated computer-based system to support nicotine dependence treatment in primary care. Nicotine Tob Res 2005;7(Suppl 1):S57-66.

10. Results from the 2012 NAQC Annual Survey of Quitlines. Phoenix, AZ: North American Quitline Consortium, 2013.

11. Kraft MR, Androwich I. Interactive voice response technology: a tool for improving healthcare. Nurs Inform 2012;2012:224.

12. McNaughton B, Frohlich J, Graham A, et al. Extended interactive voice response telephony (IVR) for relapse prevention after smoking cessation using varenicline and IVR: a pilot study. BMC Public Health 2013;13:824.

13. Blankers $M$, Smit ES, van der Pol $P$, et al. The missing=smoking assumption: A fallacy in internet-based smoking cessation trials? Nicotine Tob Res 2015. [Epub ahead of print 5 Mar 2015].

14. Asparouhov T. Muthen B. Multiple Imputation with Mplus. Technical Report. 2010. http://www.statmodel.com

15. Zbikowski SM, Jack LM, McClure JB, et al. Utilization of services in a randomized trial testing phone- and web-based interventions for smoking cessation. Nicotine Tob Res 2011;13:319-27.

16. Hughes JR, Keely JP, Niaura RS, et al. Measures of abstinence in clinical trials: issues and recommendations. Nicotine Tob Res 2003;5:13-25.

17. Orleans CT, Schoenbach VJ, Wagner EH, et al. Self-help quit smoking interventions: effects of self-help materials, social support instructions, and telephone counseling. J Consult Clin Psychol 1991;59:439-48.

18. McAfee TA, Bush T, Deprey TM, et al. Nicotine patches and uninsured quitline callers. A randomized trial of two versus eight weeks. Am J Prev Med 2008;35:103-10.
19. Bush TM, McAfee T, Deprey M, et al. The impact of a free nicotine patch starter kit on quit rates in a state quit line. Nicotine Tob Res 2008;10:1511-16.

20. McDaniel AM, Hudson D, Stratton R, et al. Pilot Study of a Technology-Enhanced Relapse Prevention Intervention. Poster presented at 10th Annual Meeting of the Society for Research on Nicotine and Tobacco, Scottsdale, AZ, 2004.

21. Kroenke K, Spitzer RL, Williams JB. The Patient Health Questionnaire-2 validity of a two-item depression screener. Med Care 2003;41:1284-92.

22. Hughes JR, Hatsukami D. Signs and symptoms of tobacco withdrawal. Arch Gen Psychiatry 1986;43:289-94.

23. Cohen S, Williamson G. Perceived stress in a probability sample of the United States. In: Spacapam S, Oskamp S, eds. The social psychology of health: Claremont symposium on applied social psychology. Newbury Park, CA: Sage, 1988:31-67.

24. Rubin DB. Multiple imputation for nonresponse in surveys. New York: John Willey \& Sons, 1987.

25. Raghunathan TE, Lepkowski JM, Van Hoewyk J, et al. A multivariate technique for multiply imputing missing values using a sequence of regression models. Surv Methodol 2001;27:85-96.

26. Senn S. Testing for baseline balance in clinical trials. Stat Med 1994;13:1715-26.

27. Javitz HS, Zbikowski SM, Deprey M, et al. Cost-effectiveness of varenicline and three different behavioral treatment formats for smoking cessation. Trans/ Behav Med 2011;1:182-90.

28. Zbikowski SM, Hapgood J, Smucker BS, et al. Phone and web-based tobacco cessation treatment: real-world utilization patterns and outcomes for 11,000 tobacco users. J Med Internet Res 2008;10:e41.

29. Zhu SH, Lee M, Zhuang YL, et al. Interventions to increase smoking cessation at the population level: how much progress has been made in the last two decades? Tob Control 2012;21:110-18.

30. Stead LF, Perera R, Lancaster T. A systematic review of interventions for smokers who contact quitlines. Tob Control 2007;16 (Suppl 1):i3-8.

31. Bush T, Zbikowski SM, Mahoney L, et al. State quitlines and cessation patterns among adults with selected chronic diseases in 15 states, 2005-2008. Prev Chronic Dis 2012;9:E163.

32. Zhou X, Nonnemaker J, Sherrill B, et al. Attempts to quit smoking and relapse: factors associated with success or failure from the ATTEMPT cohort study. Addict Behav 2009;34:365-73.

33. Hebert KK, Cummins SE, Hernandez S, et al. Current major depression among smokers using a state quitline. Am J Prev Med 2011;40:47-53.

34. SRNT Subcommittee on Biochemical Verification. Biochemical verification of tobacco use and cessation. Nicotine Tob Res 2002:4:149-59. 